\title{
Hybrid Wavelet Transform Based Melanoma Identification Using Ensemble of Machine Learning Algorithms
}

\author{
Sudeep D. Thepade, Gaurav Ramnani and Shubham Mandhare \\ Computer Engineering Dept., Pimpri Chinchwad College of Engineering, SPPU, Maharashtra, India
}

Received 15th of May 2020; accepted 24th of July 2020

\begin{abstract}
Traditional methods of disease diagnosis can be time-intensive, error prone and invasive to the subject. These methods are also prejudiced by the doctor's subjectivity. These issues can be resolved by using automated diagnosis methods. There is a considerable dearth of medical experts today, especially in the rural areas. The use of computing technology may help to assist in the diagnostic process. This paper proposes the utilization of computers to detect melanoma skin cancer. Melanoma skin cancer can be fatal, especially in its later stages. However, it shows a high recovery rate when it is detected in its early stages. Considering the lack of medical professionals, early diagnosis of melanoma may be tried using machine learning algorithms. This paper explores hybrid wavelet transform based melanoma identification using ensemble of machine learning algorithms. The hybrid wavelet transform is produced using Discrete Cosine Transform and Haar Wavelet Transform as its components. The sizes of both components are varied from $4 \times 4$ to $128 \times 128$ to obtain the hybrid wavelet transorm. Experimentation performed on the transformed dermoscopy skin images with machine learning algorithms and their ensembles gives rise to a total of 196 variations. Overall, if the average of the metrics accuracy, sensitivity and specificity is considered, the SVM algorithm using the hybrid transform of Haar 8x8 and DCT 64x64 gives the best performance, followed by the SVM algorithm using hybrid transform of Haar $128 \times 128$ and DCT $4 \times 4$. The improvised performance with reduced feature vector size, obtained by merging of two transforms, to generate hybrid transform is the major contribution of the proposed method.
\end{abstract}

Key Words: Melanoma Skin Cancer, Machine Learning, Ensemble, Dermoscopy Skin Images, Image Transforms, Feature Extraction

\section{Introduction}

Nowadays medical professionals highly rely on various automated detection techniques to assist them as these methods have proven very helpful for early detection of diseases. Earlier X-ray, ultrasound, MRI and other Computer Aided Diagnosis (CAD) systems were used for a faster identification. These automated systems are mainly used in the cases of skin cancer where early detection is of utmost importance and can be life altering.

Skin cancer is one of the most preventable cancer, it is also the most common cancer diagnosis found with over two million people diagnosed every year across the world. The ultraviolet radiation (sun's UV

Correspondence to: sudeepthepade@gmail.com

Recommended for acceptance by Angel D. Sappa

https://doi.org/10/5565/rev/elcvia.1236

ELCVIA ISSN: $1577-5097$

Published by Computer Vision Center / Universitat Autonoma de Barcelona, Barcelona, Spain 
rays) is the most common cause of skin cancers. Melanoma, which is a type of skin cancer, has been rising severely in the world for almost last 2-3 decades. It is observed that this type of skin cancer is 20 times more common in white people. People having potential genetic syndromes have a higher risk and need to regularly visit their dermatologist as a precaution.

Advanced machine learning algorithms and image processing techniques have been used in the automated systems which provide an efficient and effective way for skin cancer identification. The traditional methods were highly time consuming which involved an experienced doctor to manually go through the images to identify them with help of identification techniques like ABCDE rules, and as there was a lack of expertise in this field, these methods were having dire consequences. As a result there was a need for accurate systems which delivered results promptly for identification of melanoma skin cancer as the prognosis of melanoma skin cancer is good only when found immediately, but it is found that it drops precipitously when it spreads to other organs. This has given rise to different techniques and immensely advanced systems, with the help of which doctors are able to identify the symptoms early and then act on it immediately.

The U.S. Preventive Services Task Force (USPSTF) has made some conclusions stating that there is not enough proof to recommend against or for total body examination to find this skin cancerearly. Hence such automated systems are need of the hour for the doctors all around the globe to assist them in all possible situations in early identification of melanoma skin cancer.

The paper is structured in the following manner: Section 2 is where literature survey that was conducted for this paper is described. The proposed method is discussed in Section 3 while Section 4 illustrates the environment in which the experiments were conducted. Section 5 explicates the results that are obtained in the experiments and Section 6 gives concluding remarks on the same.

\section{Literature Review}

Automating the process of identifying melanoma from dermoscopy images has been previously undertaken by Patwardhana et al. [16], Tommasi et al. [21] and Yu et al. [22]. The activity of automating melanoma recognition can be considered as a categorization problem, where the algorithms are trained to classify the images as having malignant or benign melanoma.

The initial attempts at classifying melanoma involve the use of low-level features such as asymmetry, border and others [1] using the clinical view [2]. These attempts were categorical and rule-based and involved the use of naked-eye images with no pre-processing.

The succeeding attempts were led by the development of the technique of dermoscopy. These involved the use of surface level features [3], pattern analysis [4] and multiparameter linear classifiers [5]. The introduction of dermoscopy images proved to be an important step as it showed better and more accurate performance compared to naked-eye images [6].

Further attempts consisted of the use of texture-based features like RSurf [7], textural features [8] and extraction of texture features using Gray Level Co-occurrence Matrix (GLCM) [9]. Experiments were also conducted using color features like relative color approaches [10], different color spaces and their combinations [11] and a combination of color and shape geometry [12].

Researchers have also tried using transformation techniques like Local Binary Patterns (LBP) [13] and GLCM [14]. There have also been attempts involving the use of segmentation of the dermoscopy images using methods like Delaunay Triangulation [15] before classification. There have also been efforts to use various transforms like wavelet transforms [16] [24], Radon transforms [17] and discrete wavelet transforms [18]. 
Experiments have also been conducted by extracting colorimetric and geometric features from images and using these features to train machine learning classifiers like Support Vector Machines (SVM) [19], Naive Bayes [20] and kernel-based classifiers [21].

More recently, progression in deep learning technologies have allowed researchers to use various styles of neural networks like Residual Neural Networks [22] and Convolutional Neural Networks [23]. While these deep neural networks provide highly accurate classification performance, they have some major downsides as well. The training of these neural networks mandates a huge dataset, with an even balance between tuples of all classes. The training of these networks is also time consuming and computationally intensive. The configuration of these networks is also not easy, with a lot of trial and error required while tuning the parameters.

\section{Proposed Method}

Initially have the Discrete Cosine Transform [24] matrix of sizes $4 \times 4,8 \times 8,16 \times 16,32 \times 32,64 \times 64$ and $128 \times 128$ as well as Haar Wavelet Transform matrix of sizes 4x4, 8x8, 16x16, 32x32, 64x64 and 128x128. With the help of these two matrices we form the Hybrid Transform Matrix for Hybrid Transform (HT) consisting of Cosine:Haar-32:1 HT, Cosine:Haar-8:1 HT, Cosine:Haar-2:1 HT, Haar:Cosine-2:1 HT, Haar:Cosine-8:1 HT and Haar:Cosine-32:1 HT, accordingly giving an output matrix of $512 \times 512$. We can see this in figure 1 which shows us the formation of Hybrid basis matrix.

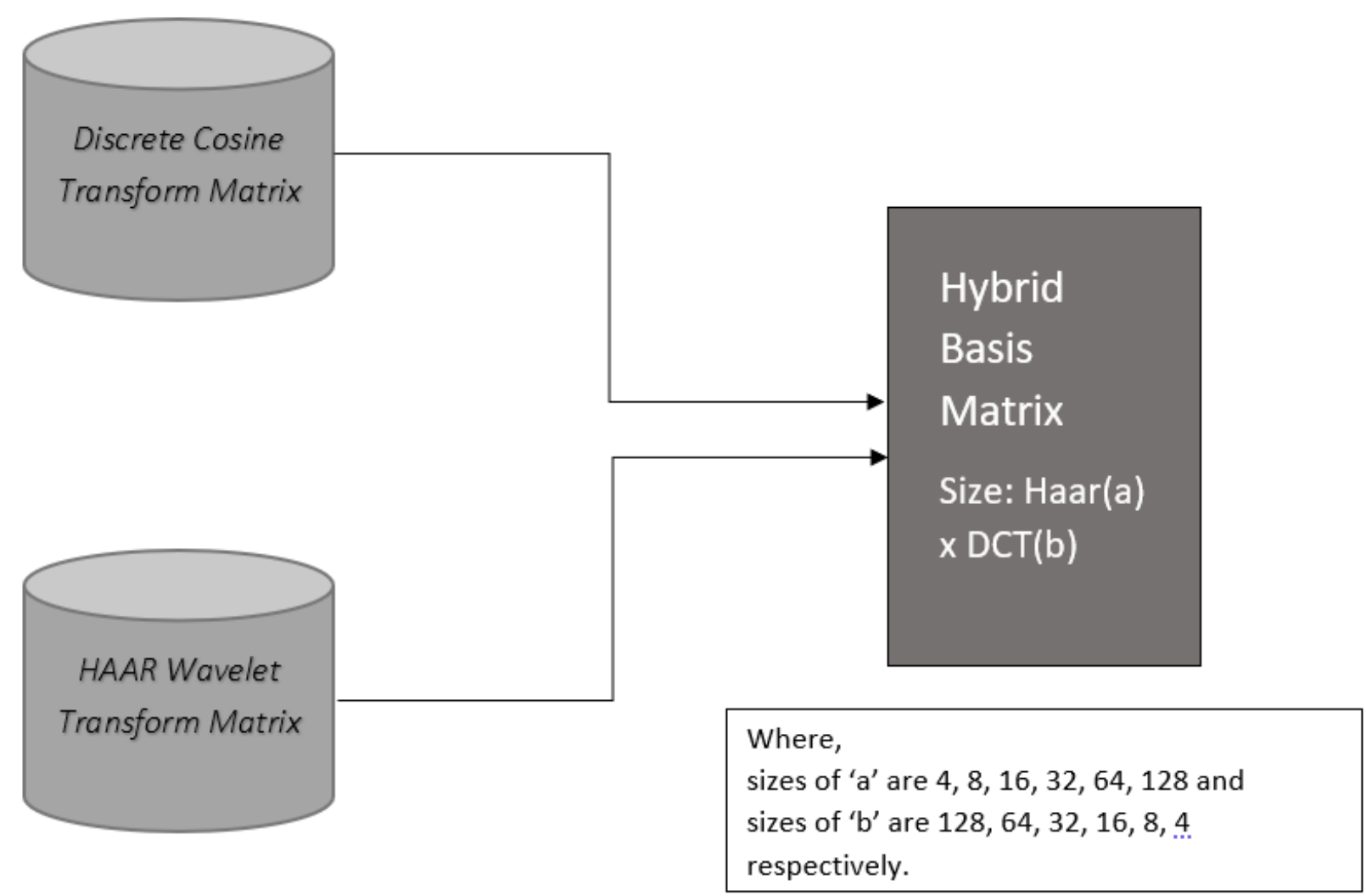

Figure 1: Phase 1- Formation of Hybrid Basis Matrix using combination of Discrete Cosine Transform and HAAR Wavelet Transform matrix

Figure 2 shows us how the Hybrid Matrix is formed in a detailed manner, where we can consider Matrix A as Haar Wavelet transform matrix and Matrix B as DCT matrix and vice versa. We can see that every element in the 1st row of Matrix A is multiplied first by the 1st column of Matrix B then with the 2nd 
column of Matrix B and so on. So, our resultant matrix width becomes MxN. In the following row we put the values of second column of Matrix A as it is and fill the remaining row by zeros. For the next row, we then shift the values ahead of the previous row and continue this till the last element of the row is filled by the value as seen in figure 2. We carry this on for the remaining rows of Matrix A and then we have our Hybrid Transform Matrix formed.

M

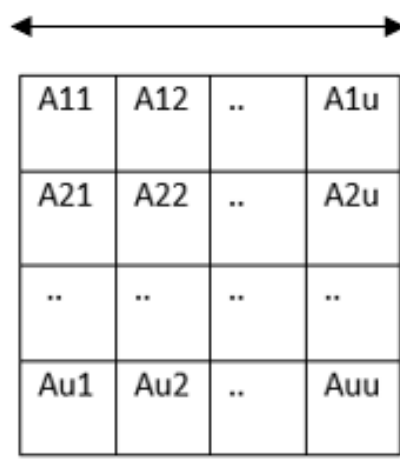

Matrix A
$\mathrm{N}$

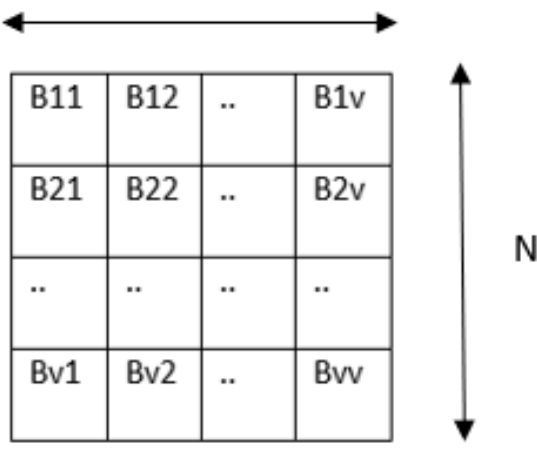

Matrix B

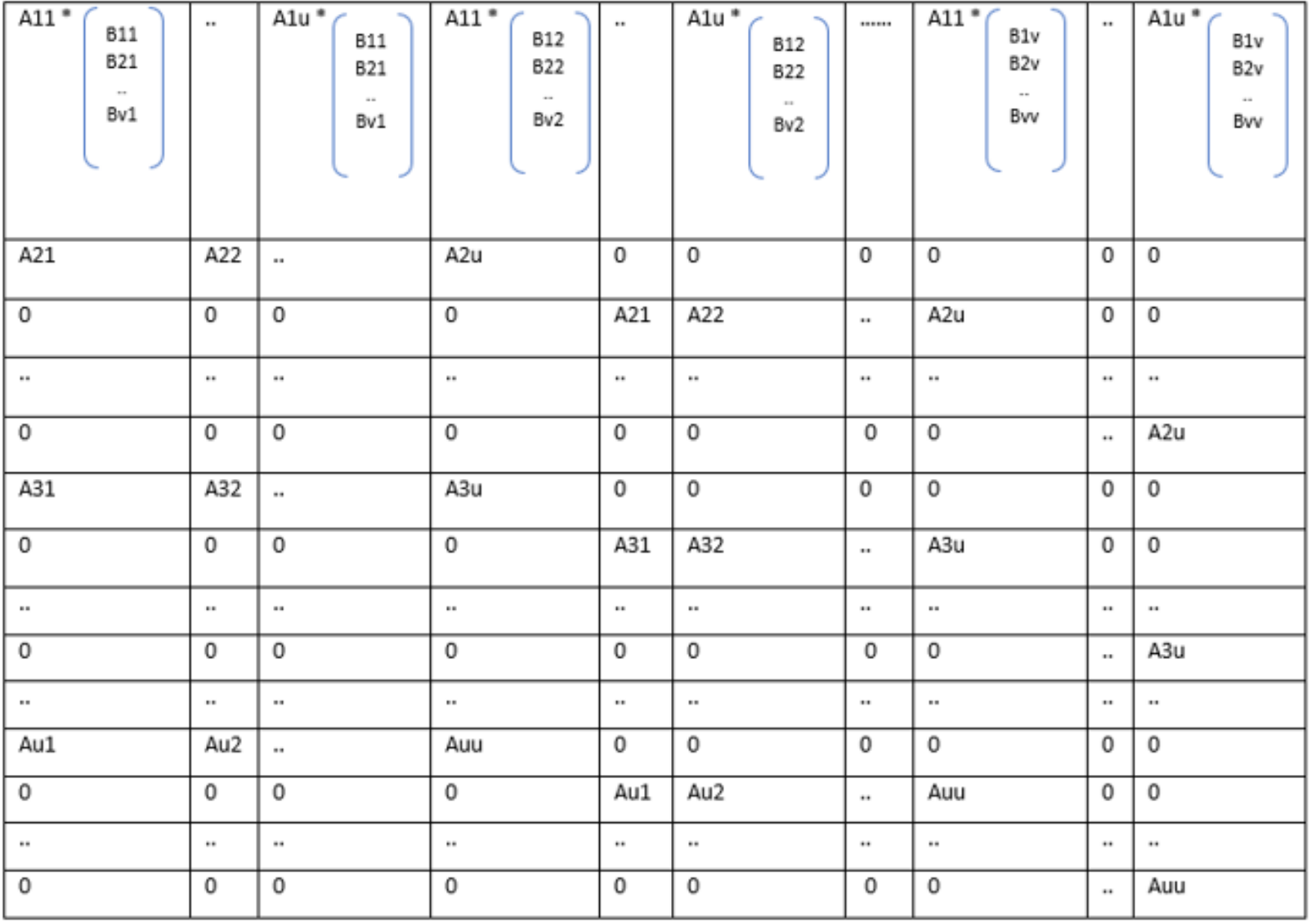

Figure 2: Generation of Hybrid Matrix

The Dermoscopy Skin images are initially read from the dataset in RGB color space. The Hybrid transform matrix which is generated with a combination of different sizes of Haar Wavelet Transform Matrix and DCT matrix is then applied on these images which give us a resultant image of size 512x512 as seen in figure 3. The Hybrid transform matrix is applied on each channel of the RGB image individually and the feature extraction process is performed separately for each channel. 


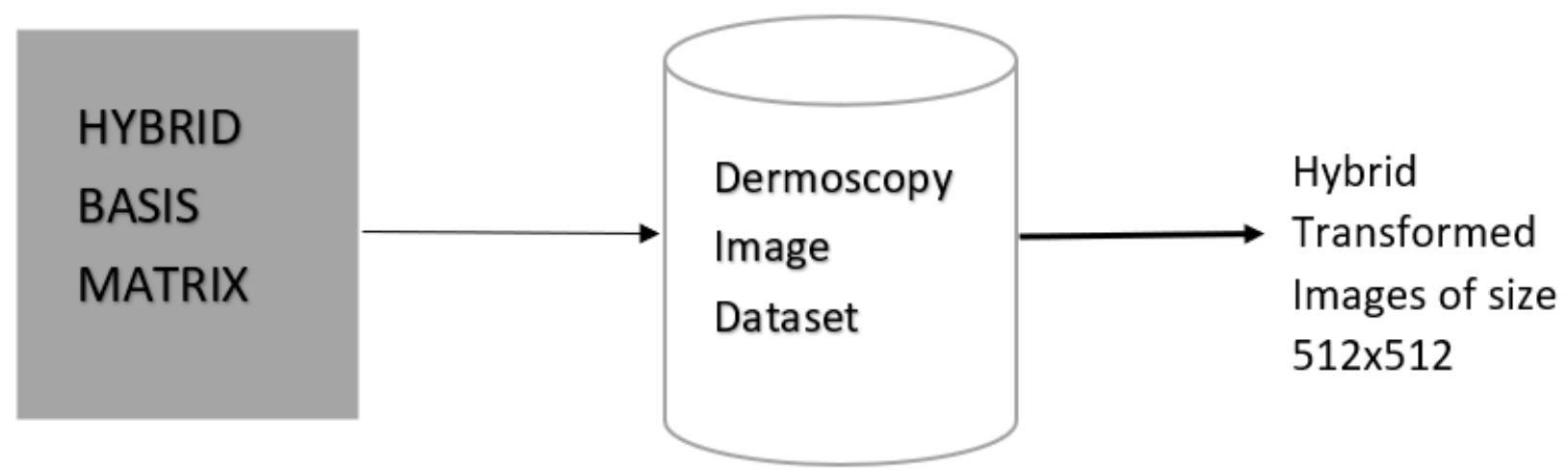

Figure 3: Phase 2 Image Transformation of Dermoscopy Skin Images using the Hybrid Transform matrix

The images transformed using the Hybrid Transform matrix then act as a training feature set for both, the individual machine learning algorithms and their ensemble combinations. These algorithms are trained with a k-cross validation of 10 folds. The trained models are then used to identify the dermoscopy skin images into malignant and benign as seen in figure 4 .

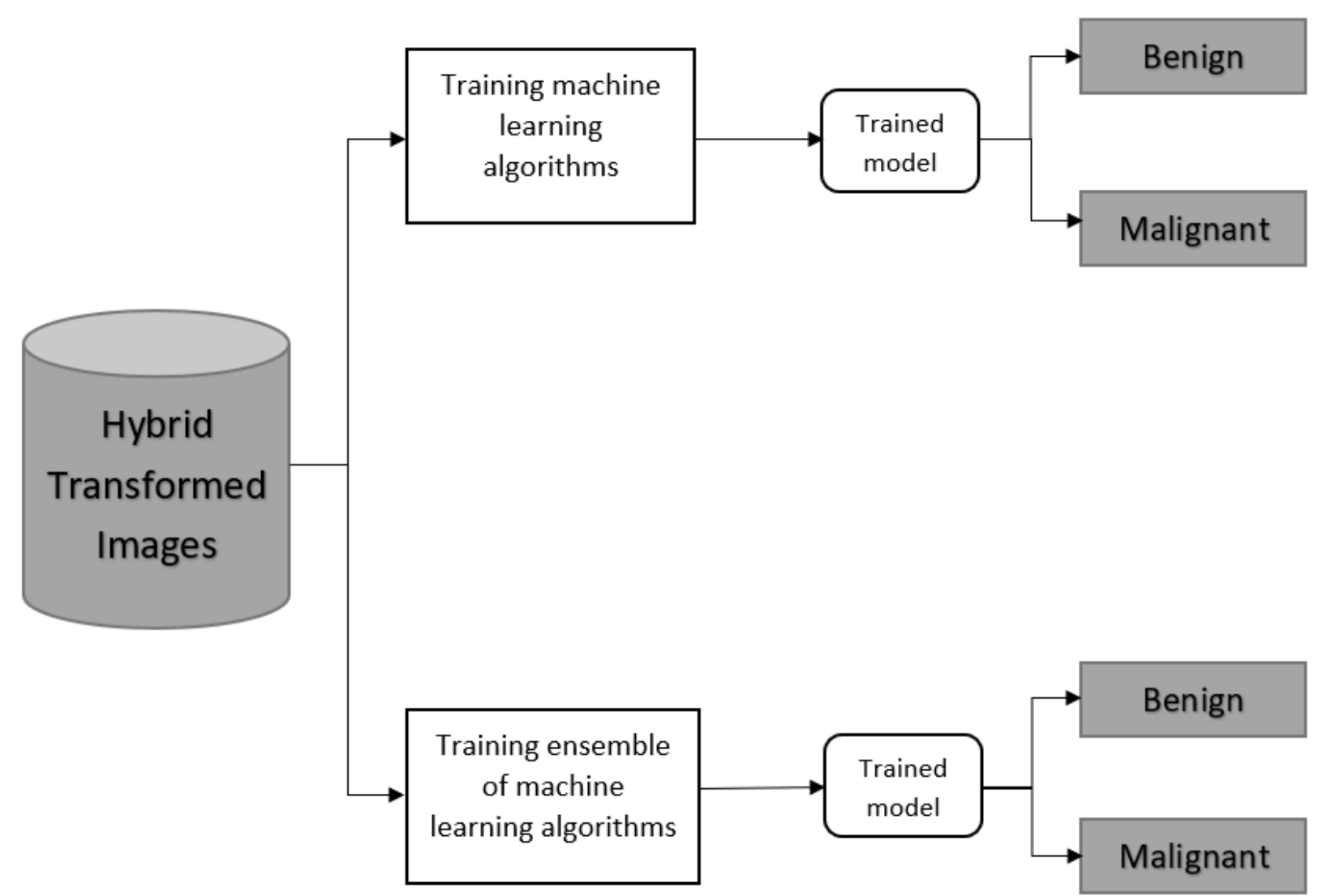

Figure 4: Phase 3- Training of machine learning algorithms and ensemble combination of machine learning algorithms on the transformed images using Hybrid TransformMatrix and then identification of melanoma skin cancer as benign or malignant. 


\section{Machine Learning algorithms used in the proposed method:}

Random Tree: When the features from the feature set are selected randomly is called a random tree. The data is always separated based on certain parameters that are obtained from decision tree features.

AD Tree: It is a classification algorithm. It consists of prediction nodes which contain a single number and decision nodes are the nodes with a predicate condition called. AD Tree follows all paths for which the decision node is true and sums up any prediction node that are traversed and, in this manner, it classifies an instance.

SVM: The main aim of this algorithm is that in an $\mathrm{N}$ (number of features) dimensional space it must find a hyperplane which classifies the data points distinctly. The objective of SVM is finding a plane having the largest distance between data points of the classes.

Random Forest: A random forest classifier is a group of multiple random decision trees. All the decision trees give a prediction for the class and the class with the maximum count of predictions becomes the model's eventual prediction.

Naïve Bayes: Naive Bayes is a collection of the categorization classifiers. It has foundation as the Bayes Theorem. The algorithm is naive because it assumes that all features are independent and equal. It predicts probabilities for each tuple belonging to specific class, with the largest probability considered as the most likely class.

Ensemble method: It is always better to use multiple models simultaneously on a single set for classification rather than just a single model. This method is called as Ensemble learning. A model is trained by using different classifiers and then the final output is the ensemble combination of them. Majority Voting logic is been used for ensemble of machine learning classifiers in proposed method.

\section{Experimentation Environment}

The Discrete Cosine Transform Matrix and Haar Wavelet Transform Matrix are used as the basic matrices for generation of Hybrid Transform matrix. The Dermoscopy Skin Image dataset used in the proposed method is taken from International Skin Imaging Collaboration (ISIC) dataset, regarded as the major prevalent collection of dermoscopy images. The dataset consists of 1000 benign and 1000 malignant images of melanoma in JPG format. The truth values of these images are provided in the form of metadata by ISIC.

Python programming language is used for the experiments in the proposed method with Waikato Environment for Knowledge Analysis (WEKA) tool for the training of different models. The k-cross validation is used for evaluation and the value of $\mathrm{k}$ is taken as 10 for the proposed method. 


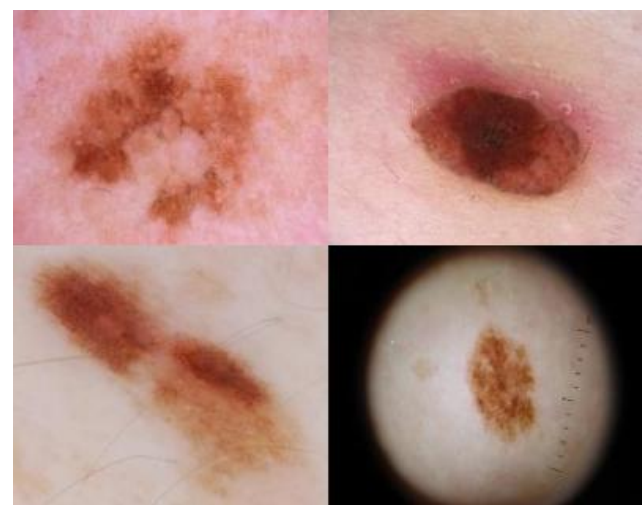

Figure 5a: Benign

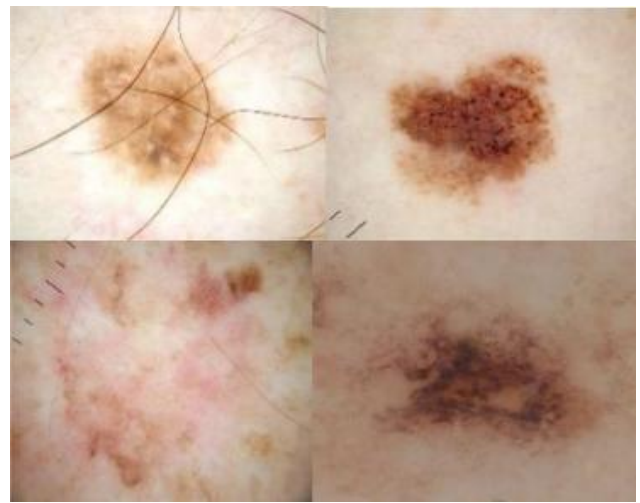

Figure 5b: Malignant

Figure 5: Image samples from ISIC Archive Dataset

The performance metrics like Accuracy (AC), Specificity (SP) and Sensitivity (SE) are used for the appraisal of performance of the proposed method of melanoma skin cancer identification. These are defined as given in equations (1), (2) and (3).

Let Rfp, Rtp, Rfn and Rtn and give the count of false positive, true positive, false negative and true negative respectively.

$$
\begin{array}{llc}
\text { Accuracy } & = & \frac{R_{t p}+R_{t n}}{R_{t p}+R_{t n}+R_{f p}+R_{f n}} \\
\text { Specificity } & = & \frac{R_{t n}}{R_{f p}+R_{t n}} \\
\text { Sensitivity } & = & \frac{R_{t p}}{R_{t p}+R_{f n}}
\end{array}
$$

Specificity is the amount of true negatives that are identified by the algorithm correctly, accuracy is the amount of both positive and negative true results, identified by the algorithm correctly and sensitivity is the amount of true positives that are identified by the algorithm correctly.

\section{$5 \quad$ Results and Discussion}

All experiments are conducted with the images in the RGB color space. To ensure uniformity while processing, all images are rescaled to a size of 512×512. The features are procured from the images using Haar wavelet transform, Discrete Cosine Transform (DCT) and the proposed hybrid transform of Haar wavelet and DCT. The fractional coefficients of these transforms are in the range from $8 \times 8$ to $64 \times 64$. The composition of the hybrid transform is also diversified by varying the sizes of both the Haar basis matrix and the DCT basis matrix while creating the hybrid basis matrix. Three machine learning algorithms and four ensembles of these machine learning algorithms are used during evaluation. These ensembles are created using the majority voting criterion. The outcomes of the experiments conducted are discussed in this section. 


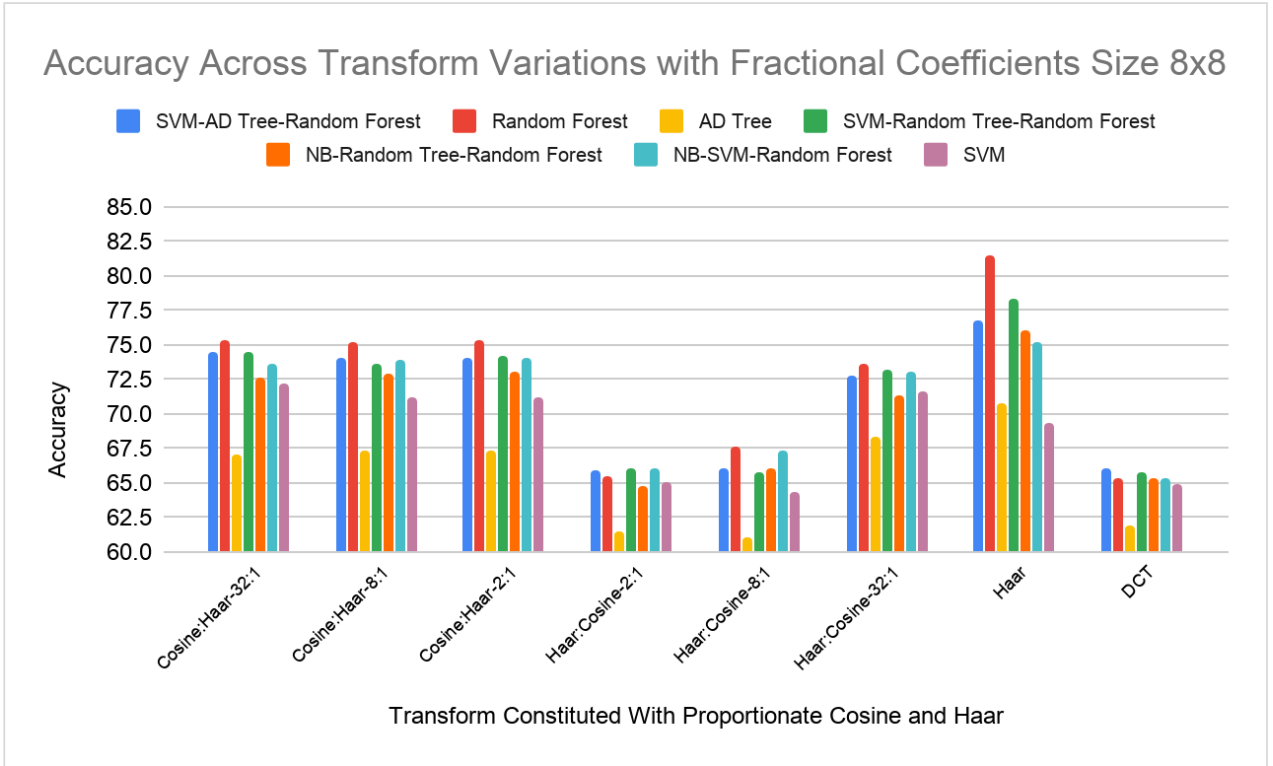

Figure 6: Performance comparison of machine learning algorithms with ensembles of algorithms in proposed feature extraction vector of transform based melanoma skin cancer detection technique for respective Haar and DCT composition variants using percentage accuracy for fractional coefficient $8 \times 8$

The capabilities comparison of the considered machine learning based algorithms and the ensembles of multiple machine learning algorithms considered together via percentage accuracy of melanoma skin cancer identification of dermoscopy images in the dataset, keeping the fractional coefficient as $8 \times 8$, is illustrated in Figure 6. It is observed that variants where the size of DCT basis matrix is higher show better performance than variants where the size of Haar basis matrix is higher. Overall, highest accuracy is attained by Random Forest for the Haar transform.

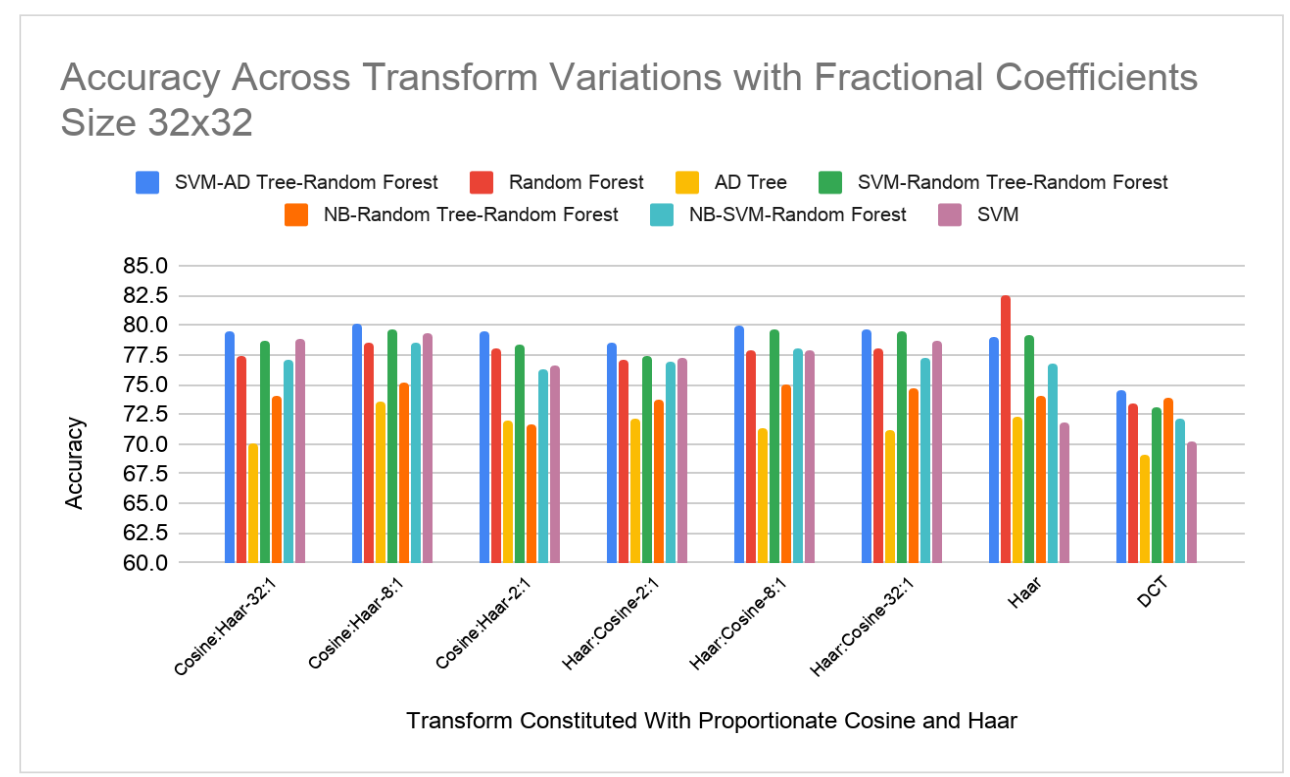

Figure 7: Performance comparison of the machine learning algorithms with ensembles of algorithms in proposed feature extraction vector of transform based melanoma skin cancer detection technique for respective Haar and DCT composition variations using percentage accuracy for fractional coefficient $32 \times 32$

Figure 7 details the capabilities collation of the considered machine learning based algorithms and the ensembles of multiple machine learning algorithms considered together using percentage accuracy of 
melanoma skin cancer identification of dermoscopy images in the dataset, keeping the fractional coefficient as $32 \times 32$. Overall, the highest accuracy is obtained by the Random Forest with the Haar transform.

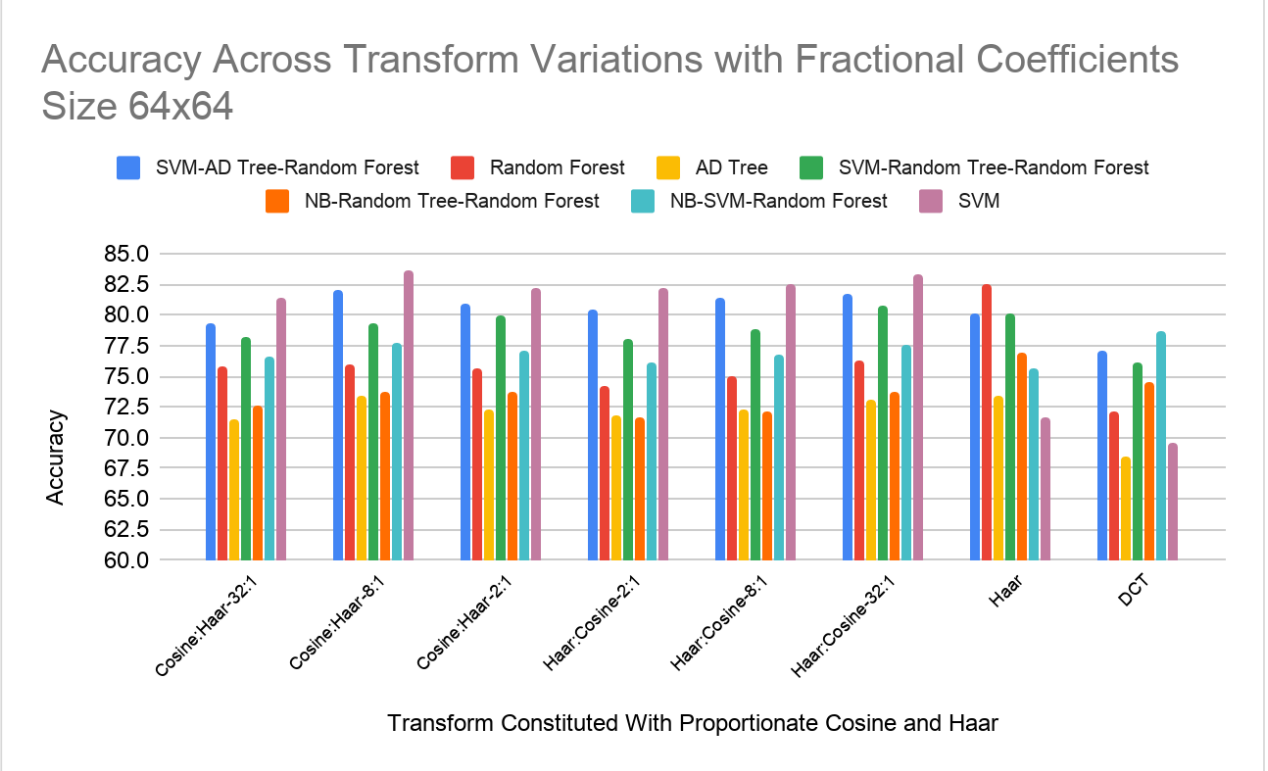

Figure 8: Performance comparison of the machine learning algorithms with ensembles of algorithms in proposed feature extraction vector of transform based melanoma skin cancer detection technique for respective Haar and DCT composition variations using percentage accuracy for fractional coefficient $64 \times 64$

Figure 8 shows the performance collation of the considered machine learning based algorithms and the ensembles of multiple machine learning algorithms through the medium of percentage accuracy of melanoma skin cancer identification of dermoscopy images in the dataset, keeping the fractional coefficient as $64 \times 64$. It can be observed that the generally the ensembles outperform the individual machine learning algorithms, however, the SVM algorithms gives best performance across all variants. The highest accuracy is achieved by SVM with the hybrid transform variant of Cosine:Haar-8:1 HT.

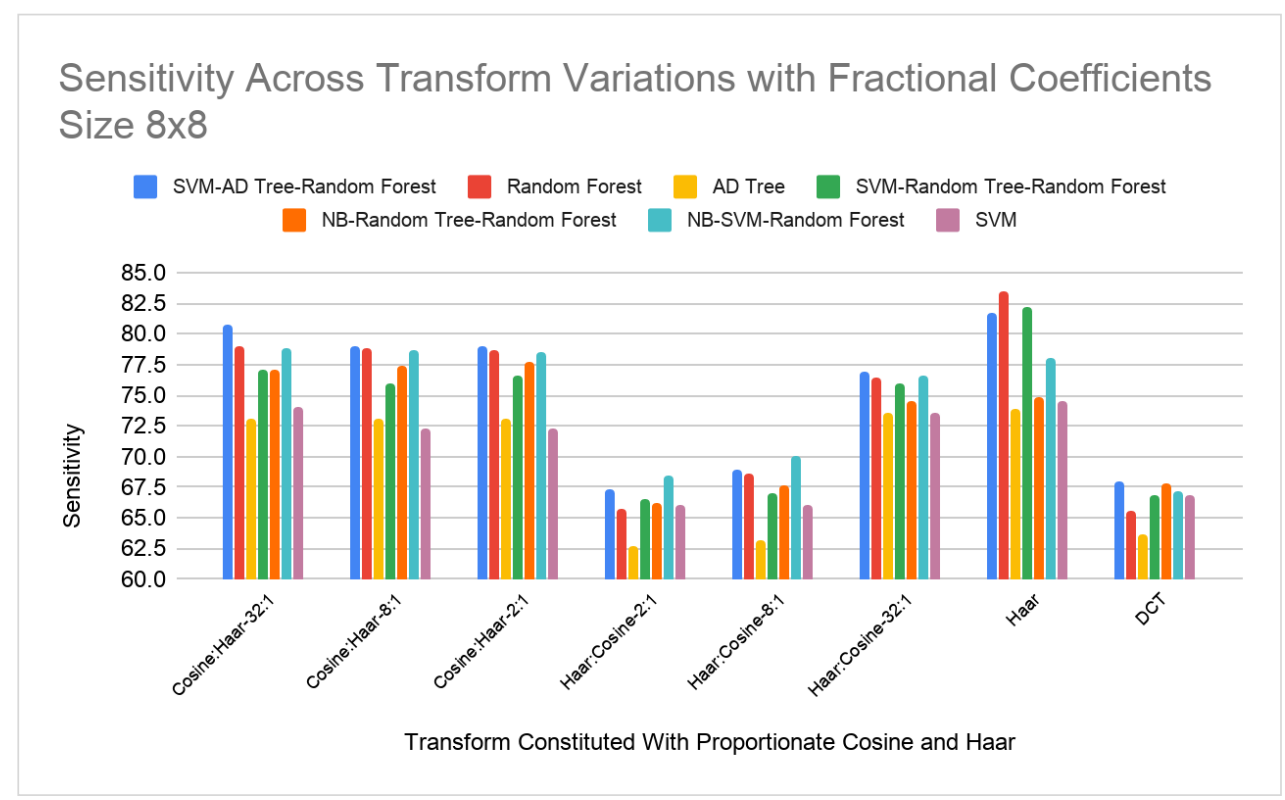

Figure 9: Performance comparison of the machine learning algorithms with ensembles of algorithms in proposed feature extraction vector of transform based melanoma skin cancer detection technique for respective Haar and DCT composition variations using percentage sensitivity for fractional coefficient $8 \times 8$ 
The capabilities comparison of the considered machine learning based algorithms and the ensembles of multiple machine learning algorithms via percentage sensitivity of melanoma skin cancer identification of dermoscopy images in the dataset, keeping the fractional coefficient as $8 \times 8$ is shown in Figure 9. It can be inferred that the hybrid transform variants where the size of DCT matrix is higher show considerably better performance compared to variants where the size of DCT matrix is smaller. Overall better sensitivity is obtained using the Random Forest for Haar transform.

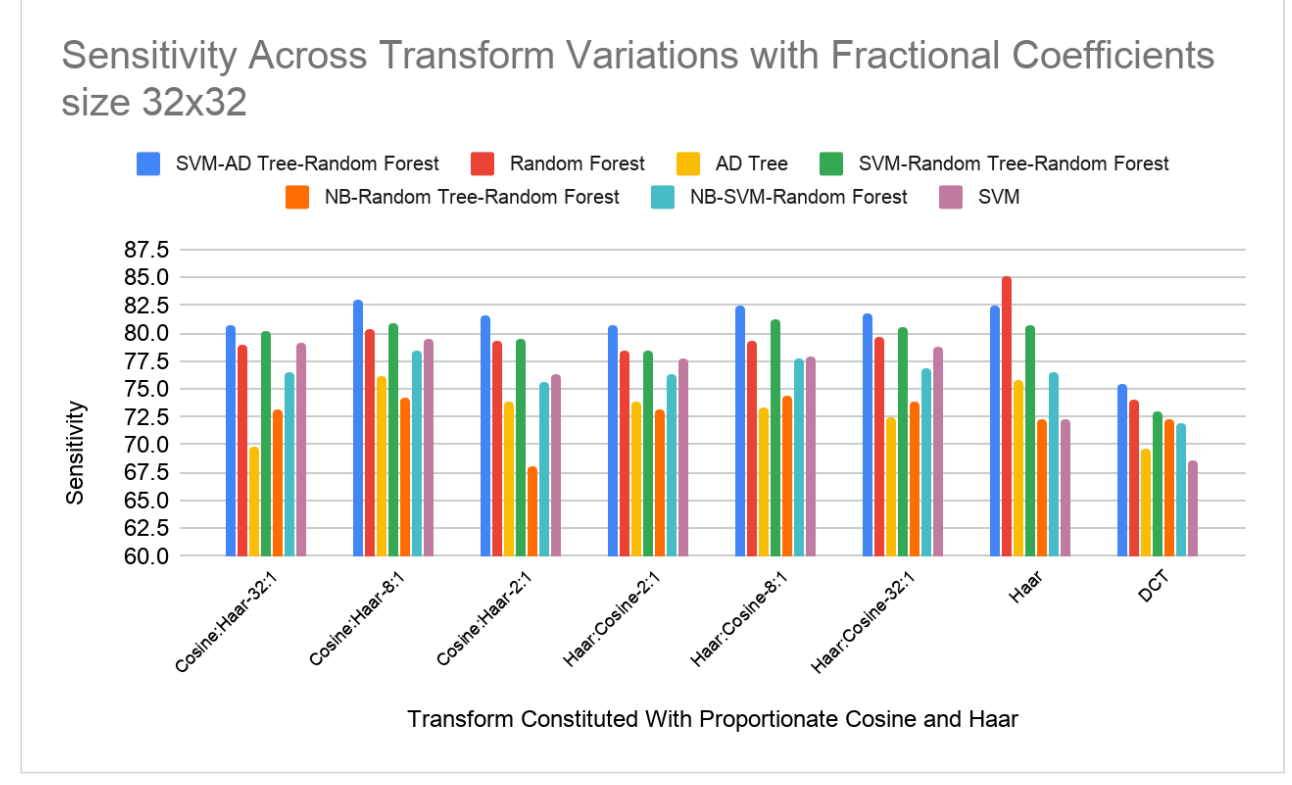

Figure 10: Performance comparison of the machine learning algorithms with ensembles of algorithms in proposed feature extraction vector of transform based melanoma skin cancer detection technique for respective Haar and DCT composition variations using percentage sensitivity for fractional coefficient $32 \times 32$

Figure 10 shows the collation of the performance of the considered machine learning based algorithms and the ensembles of multiple machine learning algorithms by means of percentage sensitivity of melanoma skin cancer identification of dermoscopy images in the dataset, keeping the fractional coefficient $32 \times 32$. Overall, highest sensitivity is obtained using the Random Forest for Haar transform.

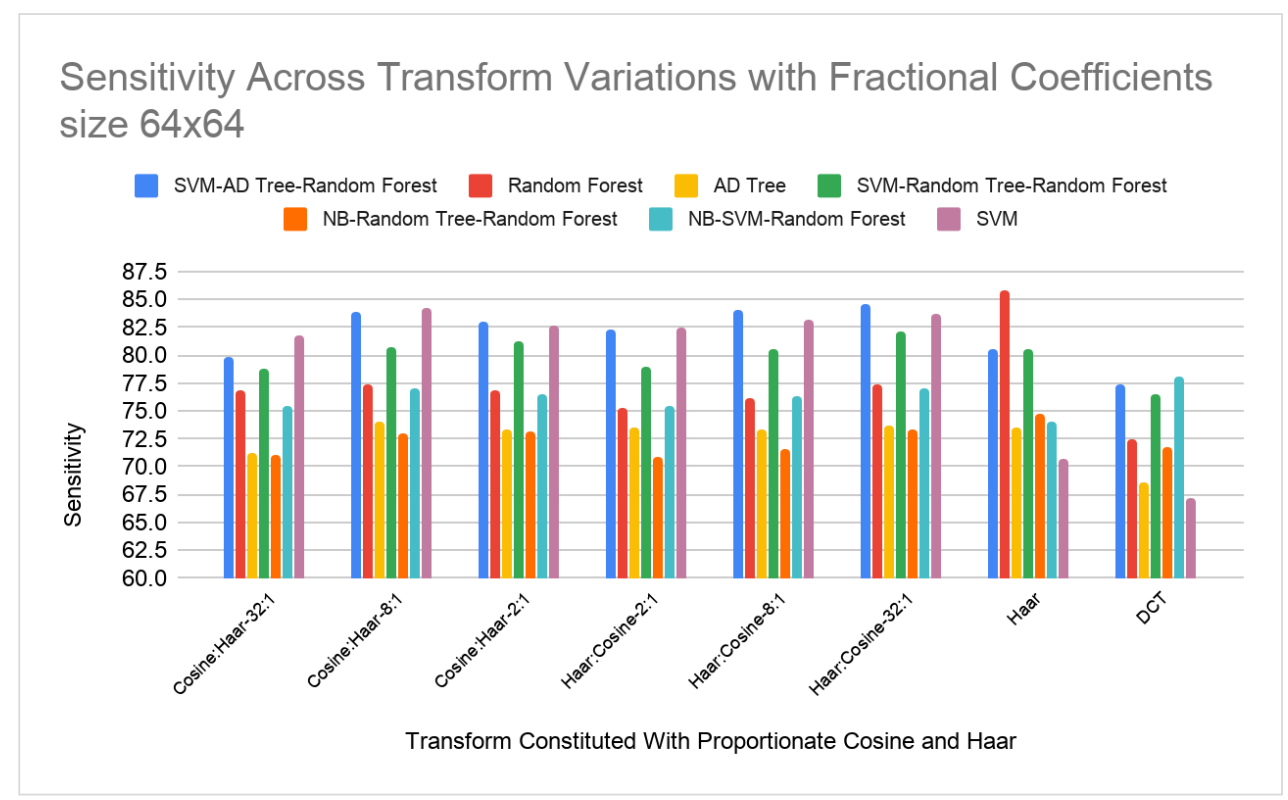

Figure 11: Performance comparison of the machine learning algorithms with ensembles of algorithms in proposed feature extraction vector of transform based melanoma skin cancer detection technique for respective Haar and DCT composition variations using percentage sensitivity for fractional coefficient $64 \times 64$ 
Figure 11 portrays the performance comparison of the considered machine learning based algorithms and the ensembles of machine learning algorithms using percentage sensitivity of melanoma skin cancer identification of dermoscopy images in the dataset, keeping the fractional coefficient $64 \times 64$. Overall, the highest sensitivity is given by the Random Forest with Haar transform.

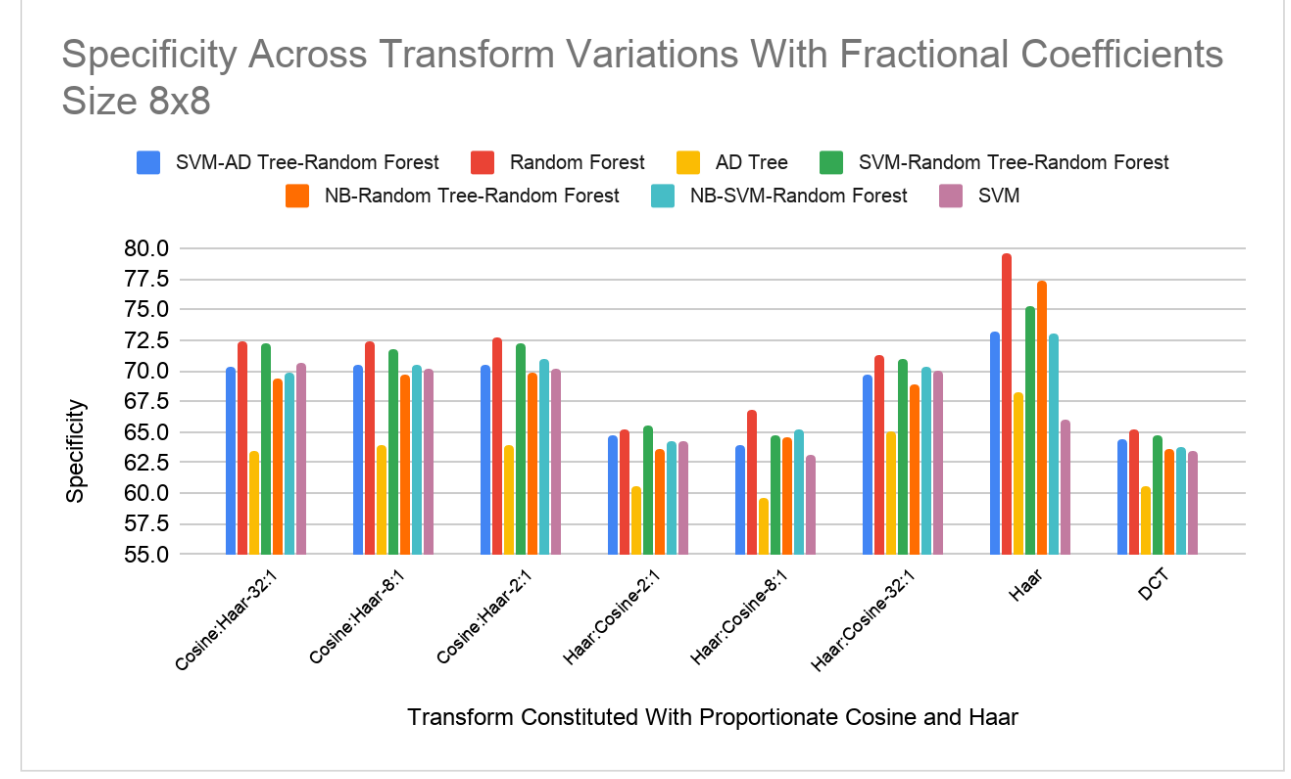

Figure 12: Performance comparison of the machine learning algorithms with ensembles of algorithms in proposed feature extraction vector of transform based melanoma skin cancer detection technique for respective Haar and DCT composition variations using percentage specificity for fractional coefficient $8 \times 8$

The performance comparison of considered machine learning based algorithms and the ensembles of multiple machine learning algorithms through specificity of melanoma skin cancer identification of dermoscopy images in the dataset, keeping the fractional coefficient $8 \times 8$ is illustrated in Figure 12. It can be seen that the hybrid transform variants having higher composition of DCT matrix outperform the variants having higher composition of Haar matrix. Overall, highest specificity is achieved by Random Forest with Haar transform.

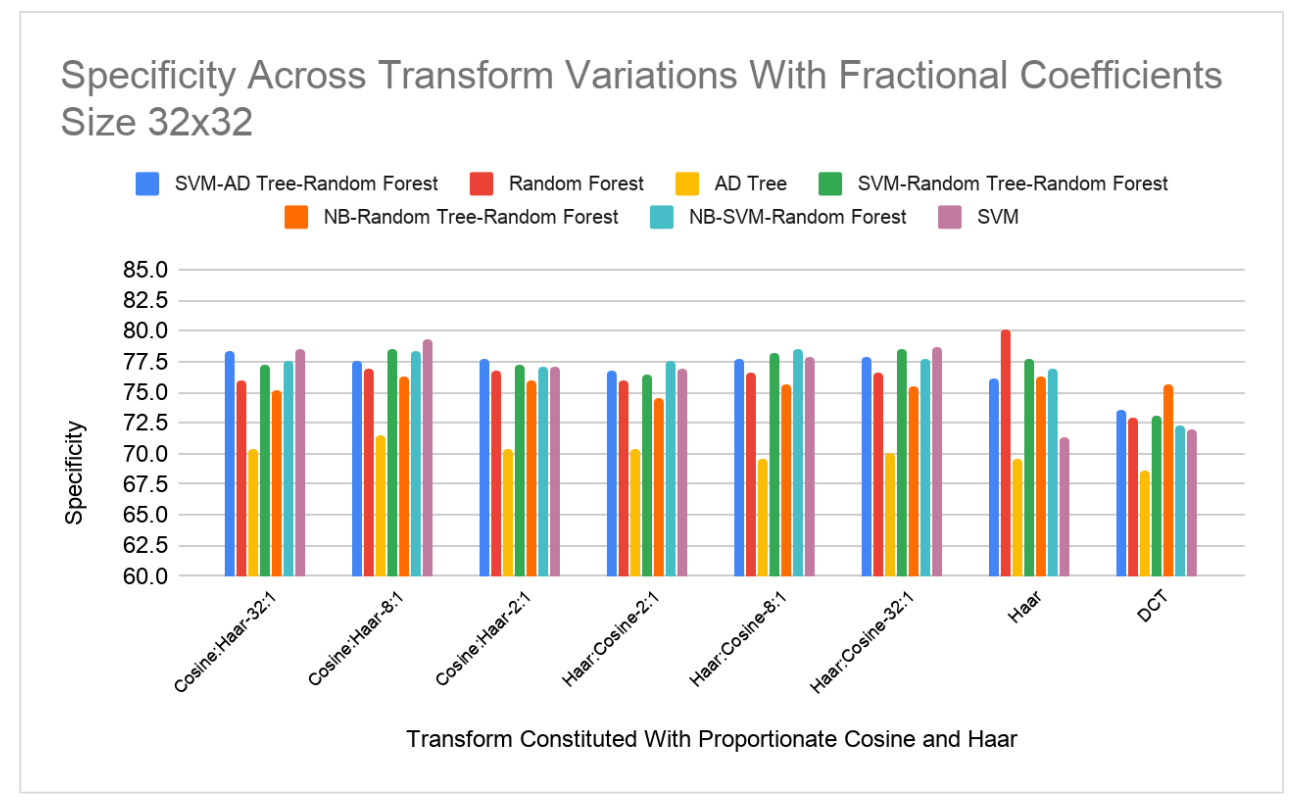

Figure 13: Performance comparison of the machine learning algorithms with ensembles of algorithms in proposed feature extraction vector of transform based melanoma skin cancer detection technique for respective Haar and DCT composition variations using percentage specificity for fractional coefficient $32 \times 32$ 
Figure 13 depicts the performance comparison of considered machine learning based algorithms and the ensembles of multiple machine learning algorithms using percentage specificity of melanoma skin cancer identification of dermoscopy images in the dataset, keeping the fractional coefficient $32 \times 32$. Overall, better specificity is obtained by Random Forest for Haar transform, followed closely by SVM with hybrid transform variant of Cosine:Haar-8:1 HT.

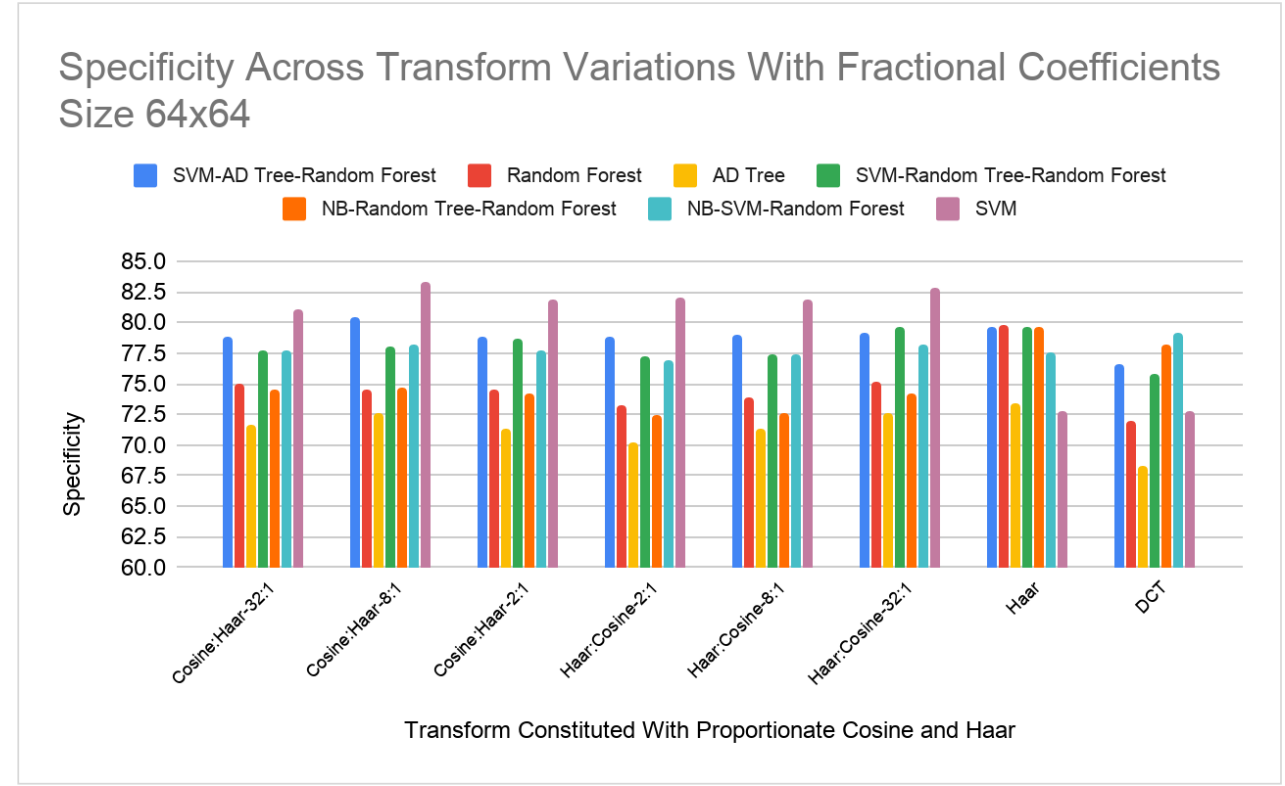

Figure 14: Performance comparison of the machine learning algorithms with ensembles of algorithms in proposed feature extraction vector of transform based melanoma skin cancer detection technique for respective Haar and DCT composition variations using percentage specificity for fractional coefficient $64 \times 64$

The performance comparison of considered machine learning based algorithms and the ensembles of multiple machine learning algorithms in virtue of percentage specificity of melanoma skin cancer identification of dermoscopy images in the dataset, keeping the fractional coefficient $64 \times 64$ is described in Figure 14. It can be seen that SVM outperforms all other algorithms, for the hybrid transform variants. Overall, highest specificity is observed using SVM with hybrid transform variant of Cosine:Haar-8:1 HT.

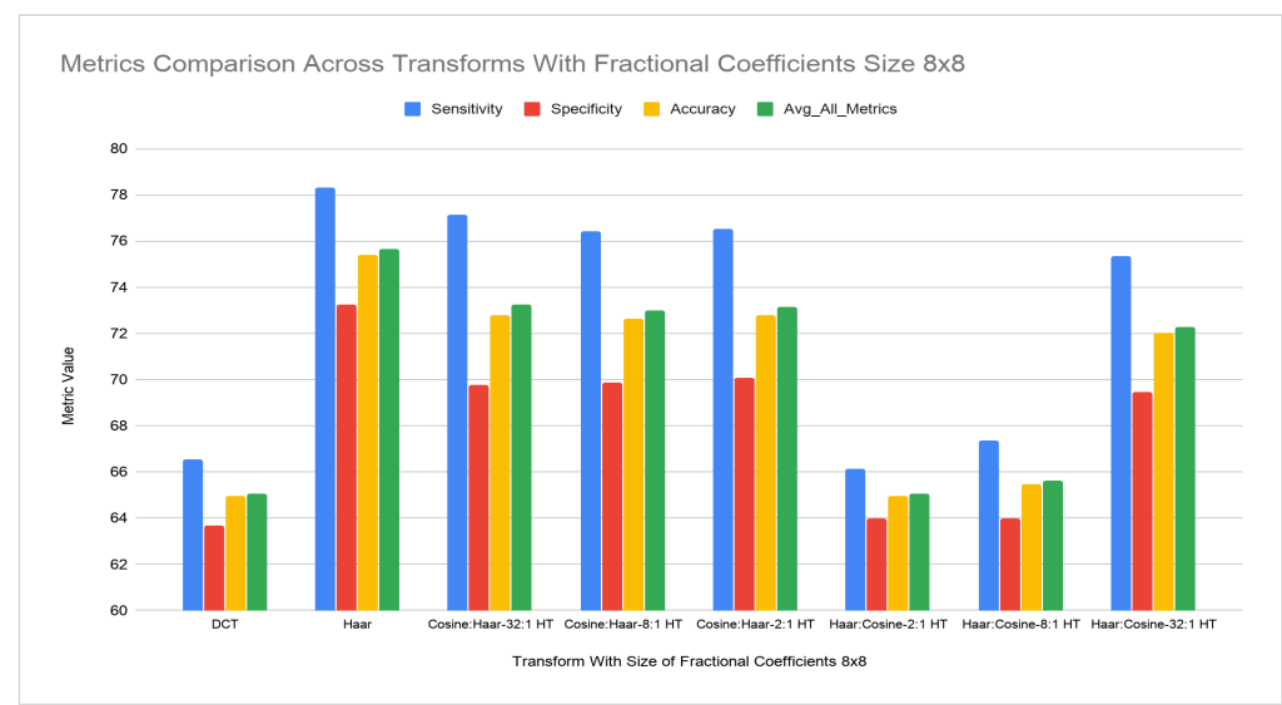

Figure 15: Performance comparison across feature extraction vectors of all transforms experimented for fractional coefficient $8 \times 8$ using accuracy, sensitivity and specificity with average values over all machine learning algorithms and ensembles of algorithms 
The performance comparison of all feature extraction vectors of all experimented transforms for fractional coefficient $8 \times 8$ across accuracy, sensitivity, specificity and the average of these three metrics is shown in Figure 15. The metric values are obtained by taking the average of all feature extraction vectors of the respective transforms. It can be inferred that Haar transform gives the highest performance for all the considered metrics.

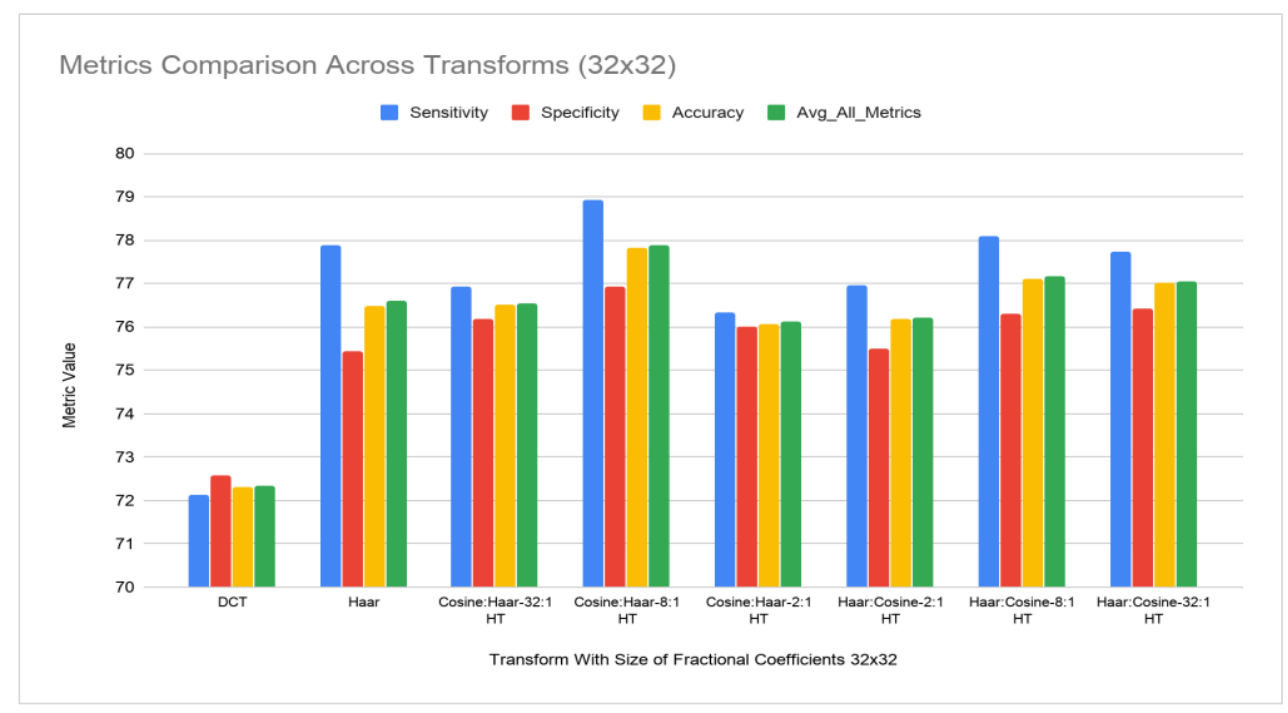

Figure 16: Performance comparison across feature extraction vectors of all transforms experimented for fractional coefficient $32 \times 32$ using accuracy, sensitivity and specificity with average values over all machine learning algorithms and ensembles of algorithms

Figure 16 portrays the performance comparison of all feature extraction vectors of all experimented transforms for fractional coefficient $32 \times 32$ over accuracy, sensitivity, specificity and the average of these three metrics. The average of the metrics of all feature extraction vectors across the respective transforms is considered. Overall, the highest performance in all metrics is obtained by the hybrid transform of Cosine:Haar-8:1 HT.

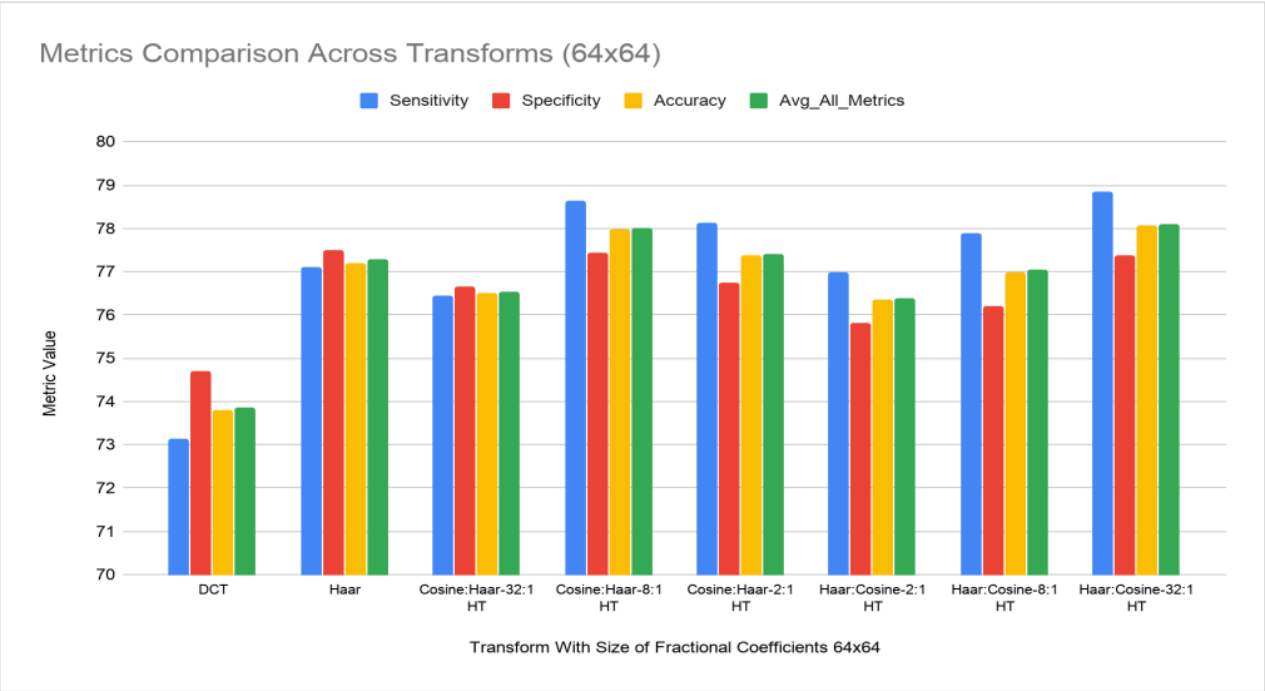

Figure 17: Performance comparison across feature extraction vectors of all transforms experimented for fractional coefficient $64 \times 64$ using accuracy, sensitivity and specificity with average values over all machine learning algorithms and ensembles of algorithms

Figure 17 describes the juxtaposition of the performance of all considered feature extraction vectors of the attempted transforms for fractional coefficient 64x64 over accuracy, sensitivity, specificity and the average 
of these three metrics. The values are obtained by taking the average of all feature extraction vectors of the respective transforms. Overall, if all metrics are given equal importance, the hybrid transform of Haar:Cosine-32:1 HT gives the best performance. It is only outperformed for the specificity metric by Haar transform.

\section{Metrics Comparison Across All Transforms}

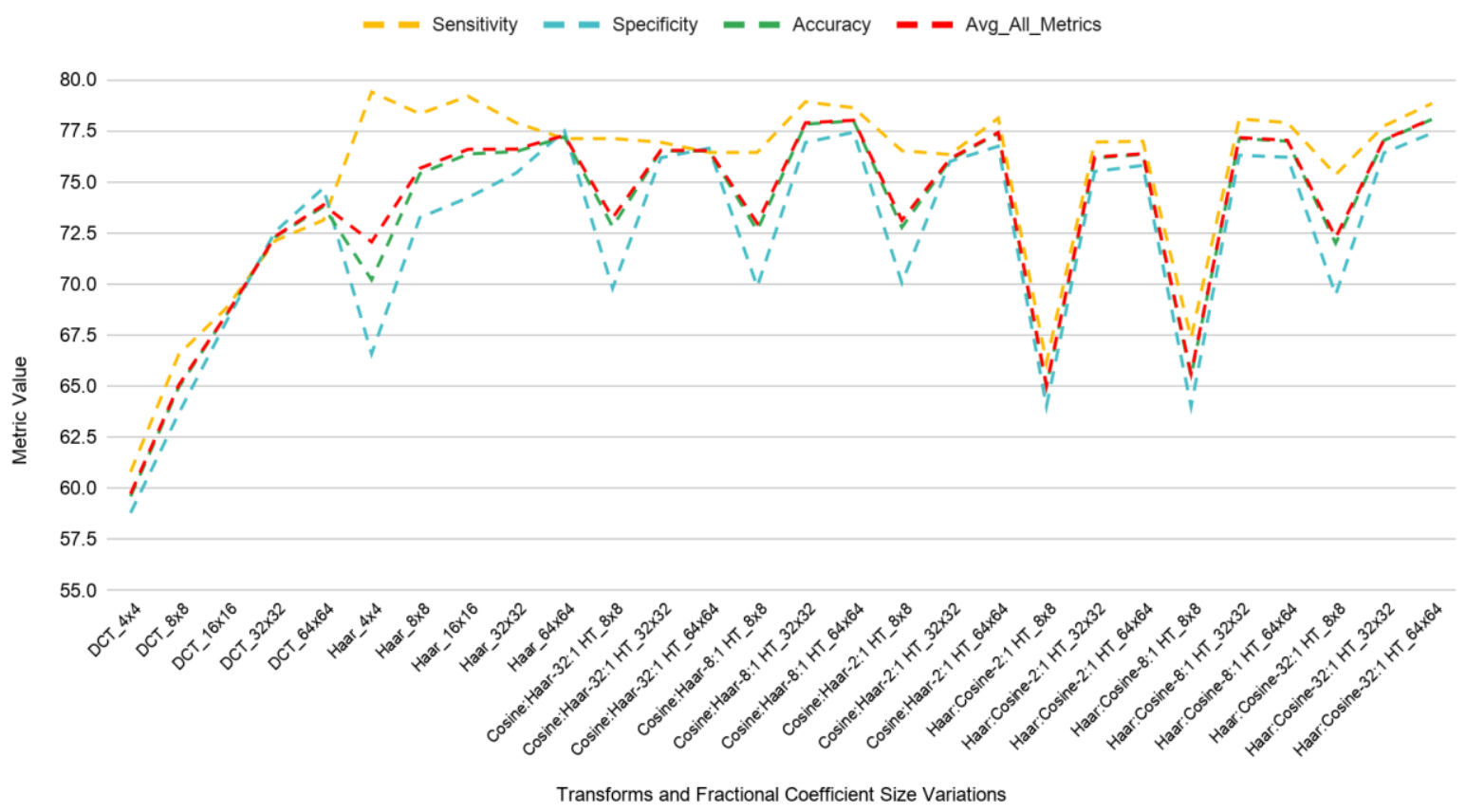

Figure 18: Performance metrics comparison across feature extraction vectors of all transforms experimented for all fractional coefficients with average values of metrics over all machine learning algorithms and ensembles of algorithms

In Figure 18, the comparison of the performance metrics (accuracy, sensitivity, specificity and the average of these three metrics) is done across all feature extraction vectors of all transforms used in the proposed method of melanoma skin cancer identification. From Figure 18, it can be inferred that if all metrics are given equal importance, the hybrid transform of Haar:Cosine-32:1 HT for the fractional coefficient size $64 \times 64$ gives the best performance, followed closely by the hybrid transform Cosine:Haar-8:1 HT for fractional coefficient size $64 \times 64$. If sensitivity is considered, the Haar transform with fractional coefficient size $4 \times 4$ performs best, while Haar transform with fractional coefficient size $64 \times 64$ gives the best performance regarding specificity. The highest accuracy is also obtained by the hybrid transform of HaarCosine-32:1 for the fractional coefficient size $64 x 64$.

Total 196 variations of the proposed wavelet based feature extraction method for melanoma skin cancer identification technique are experimented using 5 variants of Haar wavelet transform, 5 variants of Discrete Cosine Transform (DCT), 18 variations of hybrid transforms of Haar and DCT, 3 assorted machine learning algorithms (SVM, Random Forest, AD Tree) with 4 ensemble combinations. The top performing variations giving equal importance to accuracy, sensitivity and specificity are given in table 1. 


\begin{tabular}{|c|c|c|c|c|c|c|}
\hline \multirow{2}{*}{$\begin{array}{c}\text { Machine } \\
\text { Learning } \\
\text { Algorithm } \\
\text { /Ensemble }\end{array}$} & \multirow{2}{*}{$\begin{array}{c}\text { Transform Used } \\
\text { for Feature } \\
\text { Extraction }\end{array}$} & \multirow{2}{*}{$\begin{array}{l}\text { Fractional } \\
\text { Coefficient }\end{array}$} & \multicolumn{3}{|c|}{ Performance Measure } & \multirow{2}{*}{$\begin{array}{c}\text { Average of } \\
\text { Accuracy, } \\
\text { Sensitivity } \\
\text { and } \\
\text { Specificity }\end{array}$} \\
\hline & & & Sensitivity & Specificity & Accuracy & \\
\hline SVM & $\begin{array}{c}\text { Cosine:Haar- } 8: 1 \\
\text { HT }\end{array}$ & $64 \times 64$ & 84.18 & 83.23 & 83.7 & 83.70 \\
\hline SVM & $\begin{array}{c}\text { Haar:Cosine-32:1 } \\
\text { HT }\end{array}$ & $64 \times 64$ & 83.77 & 82.84 & 83.3 & 83.30 \\
\hline $\begin{array}{l}\text { Random } \\
\text { Forest }\end{array}$ & Haar & $64 \times 64$ & 85.81 & 79.84 & 82.55 & 82.73 \\
\hline $\begin{array}{l}\text { Random } \\
\text { Forest }\end{array}$ & Haar & $32 \times 32$ & 85.08 & 80.19 & 82.45 & 82.57 \\
\hline SVM & $\begin{array}{c}\text { Haar:Cosine-8:1 } \\
\text { HT }\end{array}$ & $64 \times 64$ & 83.25 & 81.88 & 82.55 & 82.56 \\
\hline $\begin{array}{l}\text { Random } \\
\text { Forest }\end{array}$ & Haar & $16 \times 16$ & 85.29 & 79.52 & 82.15 & 82.32 \\
\hline SVM & $\begin{array}{c}\text { Cosine:Haar-2:1 } \\
\text { HT }\end{array}$ & $64 \times 64$ & 82.67 & 81.84 & 82.25 & 82.25 \\
\hline SVM & $\begin{array}{c}\text { Haar:Cosine-2:1 } \\
\text { HT }\end{array}$ & $64 \times 64$ & 82.54 & 81.96 & 82.25 & 82.25 \\
\hline $\begin{array}{c}\text { SVM + AD } \\
\text { Tree + } \\
\text { Random } \\
\text { Forest }\end{array}$ & $\begin{array}{c}\text { Cosine:Haar-8:1 } \\
\text { HT }\end{array}$ & $64 \times 64$ & 83.86 & 80.51 & 82.1 & 82.16 \\
\hline $\begin{array}{c}\text { SVM + AD } \\
\text { Tree + } \\
\text { Random } \\
\text { Forest }\end{array}$ & $\begin{array}{c}\text { Haar:Cosine-32:1 } \\
\text { HT }\end{array}$ & $64 \times 64$ & 84.51 & 79.22 & 81.65 & 81.8 \\
\hline
\end{tabular}

Top 5 proposed variants with higher sensitivity
Top 5 proposed variants with higher specificity
Top 5 proposed variants with higher accuracy

Table 1: Accuracy, sensitivity and specificity considered together in the best performing variants of proposed wavelet transform based melanoma skin cancer identification technique 
If all three performance measures are given equal importance, then the best performance is observed by the Support Vector Machine (SVM) algorithm through the hybrid transform of Cosine:Haar-8:1, with fractional coefficient size $64 \times 64$, followed by the SVM algorithm using the hybrid transform of Haar:Cosine$32: 1$, with fractional coefficient size $64 \times 64$.

\section{Conclusion}

The use of computers in computer aided diagnostic techniques has proved to be great boon for humankind, allowing for quicker and more accurate diagnosis, preventing the loss of lives. These diagnostic techniques are playing a crucial role in cancer detection, especially in the areas of the world where medical experts are in short supply. This paper proposes a fresh approach to extracting features from dermoscopy images using a hybrid wavelet transform combining the qualities of two image transform techniques - Haar wavelet transform and Discrete Cosine Transform. Overall, three machine learning algorithms along with four of their ensembles are deployed while evaluating the proposed method. The performance of the constituent transform techniques along with the proposed hybrid technique is compared for melanoma skin cancer identification. The comparison of the suggested technique is performed over 196 variations using metrics like accuracy, sensitivity and specificity. The results have found that the SVM algorithm with the hybrid transform of Cosine:Haar-8:1 gives the best performance. The primary contribution of the proposed method is the improvised performance in melanoma detection, by taking the fusion of two transforms, to generate a hybrid one. It is observed that the proposed hybrid transform technique generally gives better performance compared to the individual transforms, demonstrating the worth of the advocated method.

\section{References}

[1] Hall, P. N., E. Claridge, and JD Morris Smith. "Computer screening for early detection of melanoma — is there a future?." British Journal of Dermatology 132.3 (1995): 325-338.

[2] Stoecker, William V., et al. "Nondermatoscopic digital imaging of pigmented lesions." Skin Research and Technology 1.1 (1995): 7-16.

[3] Kenet, Robert O., et al. "Clinical diagnosis of pigmented lesions using digital epiluminescence microscopy: grading protocol and atlas." Archives of dermatology 129.2 (1993): 157-174.

[4] Steiner, Andreas, Hubert Pehamberger, and Klaus Wolff. "In vivo epiluminescence microscopy of pigmented skin lesions. II. Diagnosis of small pigmented skin lesions and early detection of malignant melanoma." Journal of the American Academy of Dermatology 17.4 (1987): 584-591.

[5] Gutkowicz- Krusin, D., et al. "Can early malignant melanoma be differentiated from atypical melanocytic nevus by in vivo techniques? Part II. Automatic machine vision classification." Skin Research and Technology 3.1 (1997): 15-22.

[6] Day, Greg R., and Robert H. Barbour. "Automated melanoma diagnosis: where are we at?." Skin Research and Technology 6.1 (2000): 1-5.

[7] Majtner, Tomáš, Sule Yildirim-Yayilgan, and Jon Yngve Hardeberg. "Efficient melanoma detection using texture-based RSurf features." International Conference on Image Analysis and Recognition. Springer, Cham, 2016.

[8] Santy, Adheena, and Robin Joseph. "Melanoma Detection using Statistical Texture Distinctiveness Segmentation." International Journal of Computer Applications 127.15 (2015): 1-5.

[9] Sheha, Mariam A., Mai S. Mabrouk, and Amr Sharawy. "Automatic detection of melanoma skin cancer using texture analysis." International Journal of Computer Applications 42.20 (2012): 22-26.

[10] Stanley, R. Joe, William V. Stoecker, and Randy H. Moss. "A relative color approach to color discrimination for malignant melanoma detection in dermoscopy images." Skin Research and Technology 13.1 (2007): 62-72.

[11] Maglogiannis, Ilias, and Dimitrios I. Kosmopoulos. "Computational vision systems for the detection of malignant melanoma." Oncology reports 15.4 (2006): 1027-1032. 
[12] Abuzaghleh, Omar, Buket D. Barkana, and Miad Faezipour. "Automated skin lesion analysis based on color and shape geometry feature set for melanoma early detection and prevention." IEEE Long Island Systems, Applications and Technology (LISAT) Conference 2014. IEEE, 2014.

[13] Riaz, Farhan, et al. "Detecting melanoma in dermoscopy images using scale adaptive local binary patterns." 2014 36th Annual International Conference of the IEEE Engineering in Medicine and Biology Society. IEEE, 2014.

[14] Maurya, Ritesh, et al. "GLCM and Multi Class Support vector machine based automated skin cancer classification." 2014 International Conference on Computing for Sustainable Global Development (INDIACom). IEEE, 2014.

[15] Pennisi, Andrea, et al. "Skin lesion image segmentation using Delaunay Triangulation for melanoma detection." Computerized Medical Imaging and Graphics 52 (2016): 89-103.

[16] Patwardhan, Sachin V., Atam P. Dhawan, and Patricia A. Relue. "Classification of melanoma using tree structured wavelet transforms." Computer methods and programs in biomedicine 72.3 (2003): 223-239.

[17] Jadhav, Dattatray V., and Raghunath S. Holambe. "Feature extraction using Radon and wavelet transforms with application to face recognition." Neurocomputing 72.7-9 (2009): 1951-1959.

[18] Hamad, Asmaa, et al. "Feature extraction of epilepsy EEG using discrete wavelet transform." 2016 12th international computer engineering conference (ICENCO). IEEE, 2016.

[19] Alquran, Hiam, et al. "The melanoma skin cancer detection and classification using support vector machine." 2017 IEEE Jordan Conference on Applied Electrical Engineering and Computing Technologies (AEECT). IEEE, 2017.

[20] Li, Lin, et al. "Automatic diagnosis of melanoma using machine learning methods on a spectroscopic system." BMC medical imaging 14.1 (2014): 36.

[21] Tommasi, Tatiana, Elisabetta La Torre, and Barbara Caputo. "Melanoma recognition using representative and discriminative kernel classifiers." International Workshop on Computer Vision Approaches to Medical Image Analysis. Springer, Berlin, Heidelberg, 2006.

[22] Yu, Lequan, et al. "Automated melanoma recognition in dermoscopy images via very deep residual networks." IEEE transactions on medical imaging 36.4 (2016): 994-1004.

[23] Yuan, Yading, Ming Chao, and Yeh-Chi Lo. "Automatic skin lesion segmentation using deep fully convolutional networks with jaccard distance." IEEE transactions on medical imaging 36.9 (2017): 1876-1886.

[24] Sudeep D. Thepade, Shubham Mandhare. "Machine Learning Based Identification of Melanoma Skin Cancer Using Fractional Coefficients of Cosine Transformed Dermoscopy Images". International Journal of Advanced Science and Technology, Vol. 29, no. 05, Apr. 2020, pp. 1190 -2 